\title{
Riccati Techniques, Discrete Oscillation and Conjugacy Criteria for Fourth Order Nonlinear Difference Equations
}

\author{
Shirmila Premkumari. K
}

Abstract: We discuss the discrete Oscillatory properties of Fourth order non linear difference equation.

$$
\Delta\left(\frac{1}{r_{3}(n)}\left(\Delta\left(\frac{1}{r_{2}(n)}\left(\Delta\left(\frac{1}{r_{1}(n)}(\Delta x(n))^{\alpha_{1}}\right)\right)^{\alpha_{2}}\right)^{\alpha_{3}}\right)\right)+q(n) f(x[g(n)])=0,
$$

wherea $>1$. In particular we establish the discrete oscillation using Riccati Techniques and Conjugacy criteria. The Proofs of all the results in this paper are based on the Riccati technique.

Keywords : Conjugacy criteria, Discrete oscillation, Fourth order difference equation, Riccati techniques.

\section{INTRODUCTION}

Consider the fourth order non linear difference equation

$$
\mathbb{M}_{4} x(n)+q(n) f(x[g(n)])=0, n \in \mathbb{N}=\{0,1,2, \ldots \ldots\}
$$

(1)

$$
\text { where }
$$$$
\mathbb{M}_{0} x(n)=x(n), \mathbb{M}_{\mathbb{K}} x(n)=\frac{1}{r_{\mathbb{K}(n)}}\left(\Delta \mathbb{M}_{\mathbb{R}-1} x(n)\right)^{\alpha K} \text {, }
$$

$\mathbb{K}=1,2,3, \ldots \operatorname{mox}(2)$

$\mathbb{M}_{4} X(n)=\Delta \mathbb{M}_{a} X$ (and $\Delta$ is the forward difference operator. It is known, see [Tipler, 1978], that the most of the basic oscillatory properties of the linear differential equation $(P(t) y)^{s}+q(t) y=0$

can be extended (generalized and discretized) to the difference equation

\section{$\Delta\left(p_{\mathrm{k}} \psi\left(\Delta \mathrm{y}_{\mathrm{K}}\right)\right)+q_{\mathrm{K}} \psi\left(\mathrm{y}_{\mathrm{K}+1}\right)=0(4)$}

where $p k$ and $q k$ are real-valued sequences defined on with $r_{K}=0$ and, from those we mention the possibility to use the Riccati Technique in the Oscillatory theory that is based on the equivalence between a non oscillatory and a solvability of the generalized Riccati difference equation. This technique is used for establishing the so called Hart man-wintner lemma for equation (3) with $r(t) \equiv 1$, see [Kwong et al., ]. Here we shall assume that

(i) $p_{i}(n), q(n): \mathcal{N} \rightarrow \mathcal{R}^{+}=(0, \infty), i=1,2,3$

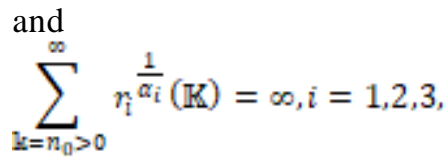

\author{
$\mathcal{G}(n): \mathcal{N} \rightarrow \mathcal{R},\{\mathcal{G}(n)\} \lim _{n \rightarrow \infty} \mathcal{G}(n)=\infty$ \\ $\mathcal{F} \in \mathcal{C}(\mathcal{R}, \mathcal{R}), X \mathcal{F}(X)>0$, and $\mathcal{F}^{\prime}(\chi) \geq$ \\ (ii) 0 , for $\chi \neq 0$
}

(iii) $\alpha_{i_{a}} i=1,2,3$ are the ratios of positive odd integers.

The domain $D(L 4)$ of $L 4$ is defined to be the set of all function $x(n):[n x, \infty) \rightarrow \mathrm{R}, n x \geq n 0 \geq 0$ such that $\operatorname{Lj} x(n), 0$ $\leq j \leq 4$ exist on $[n x, \infty)$. A solution $\{x(n)\}$ of equation (1) is called oscillatory if for any $m \in \mathrm{N}$ there exist $m, m 2 \geq m$ such that $x(m 1) x(m 2)<0$, otherwise it is non oscillatory. equation (1) is called B-oscillatory if all its bounded solutions are oscillatory. Our approach to the discrete oscillation and conjucation of equation (1) will be based largely on a discrete version of the Riccati equation. The necessary and the sufficient condition for the oscillation of equation (1) is expressed in terms of the existence of a certain solution of the generalized Riccati difference equation which is in the summation form. Determining oscillation and non oscillation criteria for difference equation has received a great deal of attention in the last few years. See, for example the monographs [Chen and Erbe, 1989, Cheng, 1994, Dosly and Rehak, 1998b, Dosly and Rehak, 1998a, Erbe and Yan, 1992, Hooker et al., 1987, Kwong et al., , Selvaraj and Lovenia, ] and the references cited there in. This interest is motivated by the importance of difference equations is the numerical solution of differential equation. Compared to first and second order difference equations, the study of higher order equations and in particular fourth order equations of type equation (1) with $\alpha i=1, i=1,2,3$ and some $\alpha i(n) \equiv$ $1, i=1,2,3$ has received considerably less attention (see [Hooker and Patula,

1981, Patula, 1979, Selvaraj and Daphy, 2011, Taylor, 1993, Tipler, 1978, Wintner, 1951, Mary,

1987]) and the referenced cited there in.

Our purpose of this paper is to obtain some sufficient conditions for the discrete oscillation of all bounded solutions of egyation (1). And also we discuss some conjugacy criteria for the equation of type (1). 


\section{MAIN RESULTS}

Consider the following inequality $\mathbb{M}_{4} x(n)+q(n) f(X[g(n)])=0, n \in \mathbb{N}=\{0,1,2, \ldots \ldots\}$

(6)

If (6) is non oscillatory, then the solution $x n$ is said to be principal or recessive in case

$$
\sum_{n=\mathbb{N}}^{\infty} \alpha_{n} x_{n} x_{n+1}=+\infty
$$

for some $\mathbb{N} \geq$ and is said to be non-principal or dominant in case

$\sum_{n=\mathbb{N}}^{\infty} \alpha_{n} x_{n} x_{n+1}<+\infty$

Definition 2.1 (i) An interval $(m, m+1]$, is said to contain the generalized zero of a solution of (6), if $x_{m} \neq 0$ and $a_{m} x_{m} x_{m+1} \leq 0$.

(i) Equation (6) is said to be disconjugate on the discrete interval $[\mathrm{m}, \mathrm{n}]$ provided any solution of this equation has at most one generalized zero on $\left(\mathrm{m}_{0} \mathrm{~m}+1\right]$. And the solution $\mathrm{x}$ satisfying $\mathrm{xm}=0$ has no generalized zero on $\left(\mathrm{m}_{1} \mathrm{~m}+1\right]$. Otherwise (6) is said to be conjugate on $[\mathrm{m}, \mathrm{n}]$.

(ii)Equation (6) in said to be non oscillatory if there exist $\mathrm{m} \in \mathrm{N}$. such that this equation is disconjugate on $[\mathrm{m}$, $n]$ for every $n>m$.

(iii) The application of the following result is usually referred to as the Riccati technique.

Proposition 2.2 Equation (1) is non oscillatory if and only if there exist a sequence $W$ and $m \in \mathrm{N}$ such that

$$
\mathcal{R}\left[\mathcal{W}_{2}\right]=\Delta \mathcal{W}_{\mathrm{n}}+q_{\mathrm{n}}+s\left(\mathcal{W}_{2}\right.
$$

with ${\mathbb{\mathbb { W } _ { \mathrm { L } }}}+w_{\mathrm{L}}>0$ for $\mathbb{R} \geq m$, where

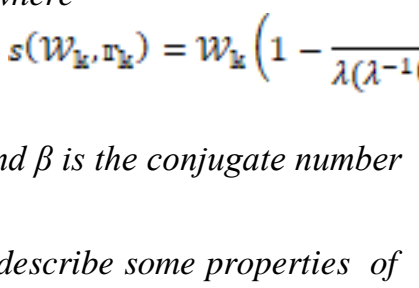

$\lambda^{-1}$ being the inverse of $\lambda$,

i.e., $\lambda^{-1}(x)=|x|^{\Phi-1}$ sgn $x$ and $\beta$ is the conjugate number of $\alpha$, ie., $\frac{1}{\alpha}+\frac{1}{\beta}=1$.

At the end of this section we describe some properties of the function, assuming $w_{\mathrm{h}}=X_{s} \mathbb{1}_{\mathrm{Wh}}=$

$s(x, y)=x\left(1-\frac{y}{\lambda\left(\lambda^{-1}(x)+\lambda^{-1}(y)\right)}\right)$

Lemma 2.3 Suppose that $\alpha>1$. The function $s(x, y)$ has the following properties:

(i) $s(x, y)$ is continuously differentiable on

$$
\mathcal{D}=\{(x, y) \in \mathcal{R} * \mathcal{R}, x \neq-y\}
$$

(ii) Let $y>0$. Then $S_{x}(x, y) \geq 0$ for $x+y>0$, where $S_{x}(x, y)=0$ if and only if $x=0$.

(iii) Let $x+y>0$. Then $S_{y}(x, y) \geq 0$, where the equality holds if and only if $x=0$.

(iv) $s(x, y) \geq 0$ for $x+y>0$, where the equality holds if and only if $x=0$.

(v) Suppose that the Sequence ( $x k, y k), K=1,2, \ldots$, is such that $x K+y K>$ Oand there exists a constant $M>0$ such that $y K \leq M$ for $K=1$, $2,3, \ldots$ Then $S(x k, y k, \infty) \rightarrow 0$ implies $x k \rightarrow 0$. Moreover, liminfyk $\geq 0$.

$$
k \rightarrow 0
$$

(vi) Let $\bar{s}(x, y)=x-s(x, y)$. Then

$\bar{S}(x, y)=\bar{S}(y, x)$ on $D$ and $\overline{S_{x}}(x, y) \geq 0$ for

$x+y>0$, where the equality holds if and only

if $y=0$. If $y \leq 1$, then

$\bar{s}(x, y)<1$ for all $x+y>0$.

\section{DISCRETE OSCILLATION AND NON OSCILLA- TION CRITERIA}

We shall be interested in using the Riccati equation along with certain averaging techniques to obtain some new discrete oscillation and non oscillation criteria.

We denote by $S$ the set of all real sequences $\mathbb{C}=\left\{\mathbb{C}_{n}\right\}_{n}^{\infty}$ such $\quad \lim _{n \rightarrow \infty} \sum_{j=0}^{n} \mathbb{C}_{j} \equiv \sum_{j=0}^{\infty}$ exists

(finite).

We shall also need the following conditions which will be imposed in the theorems to follow:

(U1)

$\lim _{n \rightarrow \infty} \sup n^{-\frac{3}{2}} \sum_{j=0}^{n} x_{j}<+\infty(U 2)$

$\operatorname{llim}_{n \rightarrow \infty} \sec \left(\frac{1}{n}\right) \sum_{j=0}^{n} \gamma_{j}<+\infty(U 3)$ (There exists $M>0$ with

$0<x_{n} \leq M$ for all $n \geq 0$. Clearly, $(A 3) \Rightarrow(A 2) \Rightarrow(A 1)$.

Theorem 3.1 Assume that (U1) holds and that (1) is non cillato $\left.\left(w_{3}\right)_{4}\right) n$ the following statements are equivalent:

(i) $\lim _{n \rightarrow \infty}\left(\frac{1}{n}\right) \sum_{i=0}^{n} \sum_{i=0}^{\prod} q_{i}$ exists (finite).

(ii) The sequence $q=\left\{q_{i}\right\}_{i=0}^{\infty}$ satisfies

$\lim _{n \rightarrow \infty} \inf \left(\frac{1}{n}\right) \sum_{\mathrm{i}=0}^{n} \sum_{\mathrm{i}=0}^{\mathbb{1}} q_{\mathrm{i}}>-\infty$

(iii) For any non oscillatory solution $x$

with $x_{n} x_{n+1}>0, n \geq \mathbb{N}$, for some $\mathbb{N} \geq 0$, the sequence $u_{n}=\frac{v_{n} \Delta x_{n}}{x_{n}}, n \geq \mathbb{N}$, satisfies

$\sum_{i=0}^{\mathbb{l}} \frac{u^{2}{ }_{i}}{u_{i}+r_{i}}<+\infty$

Proof: Clearly $(i) \rightarrow($ ii $)$. To show that $($ ii $) \rightarrow($ iii $)$ suppose to the contrary that there is a non oscillatory solution $x(n)$ of (1) such that $u_{n}=\frac{x_{n} \Delta x_{n}}{x_{n}}>-$ for $n \geq$ and 


$$
\begin{aligned}
& \sum_{i=\mathbb{N}}^{\infty} \frac{u_{i}^{2}}{u_{i}+r_{i}^{n}}=+\infty \\
& u_{n+1}+\sum_{i=N}^{n} \frac{u_{j}^{2}}{u_{j}+r_{j}}+\sum_{i=\mathbb{N}}^{\infty} q_{i}=u_{n}
\end{aligned}
$$

and therefore

$\frac{1}{n} \sum_{i=N}^{n}-U_{i+1}=\frac{1}{n} \sum_{i=N}^{n} \sum_{i=N}^{K} \frac{\tau_{i}^{2}}{{\tau_{i}+r_{j}}_{n}}+\frac{1}{n} \sum_{i=N}^{n} \sum_{i=N}^{K} q_{i}-$ $\left(\frac{n-N+1}{n}\right) u_{n}$ for $n \geq \mathbb{N}$

From (9),(11),(13) we obtain

$\lim _{n \rightarrow \infty} \frac{1}{n} \sum_{i=\mathbb{N}}^{n}\left(-u_{i+1}\right)=+\infty$

and here

$\lim _{n \rightarrow \infty} \frac{1}{n} \sum_{i=N}^{n}\left|u_{j}\right|=+\infty$

Let $\nu_{n}=\frac{{w_{n}}^{2}}{{w_{n}+v_{n}}_{n}}$ for $n \geq \mathbb{N}$. Then $\nu_{n} \geq 0$ and $\nu_{n}=0$ if and only if

$u_{n}=0$. Let $b_{n}=\frac{{w_{n}}^{2}}{\mathscr{T}_{n}}$ if $U_{n} \neq 0$ and $b_{n}=0$ if $u_{n}=0$.Then we have $r_{n} \geq b_{n}-v_{n}$ and hence

$n^{-3 / 2} \sum_{i=\mathbb{N}}^{n} x_{i} \geq n^{-\frac{3}{2}} \sum_{i=\mathbb{N}}^{n} a_{i}+n^{-\frac{3}{2}} \sum_{j=\mathbb{N}}^{n}-u_{j}$

Now from (16), ( $U 1)$ and $a n \geq 0$ it follows that

$\limsup _{n \rightarrow \infty} n^{-\frac{3}{2}} \sum_{i=\mathbb{N}}^{n}-u_{i}<\infty$

(ii) we obtain

$\limsup _{n \rightarrow \infty} n^{-\frac{3}{2}} \sum_{i=\mathbb{N}}^{n} \sum_{i=\mathbb{N}}^{n} v_{i}<+\infty$

we have

$\limsup _{n \rightarrow \infty} n^{-\frac{3}{2}} \sum_{i=N}^{n} v_{i}<+\infty$

on the other hand, from (19) there is an $M>0$ such that

$n^{-3 / 2} \sum_{i=\mathbb{N}}^{n} r_{i} \geq \frac{1}{M}\left(\frac{1}{n} \sum_{i=\mathbb{N}}^{n}\left|u_{j}\right|\right)^{2}$

So from (15)-(17) and (20) we have

$\lim _{n \rightarrow \infty} n^{-\frac{3}{2}} \sum_{i=\mathbb{N}}^{n} x_{i=1}^{n}=+\infty$

which contradicts (U1). There fore (ii) $\Rightarrow($ iii $),(i i i) \Rightarrow(i)$ Let $u$ be the sequence in (iii) and

let $B_{n}=\sum_{i=N}^{n}\left|U_{j}\right|$ then we have

$\left(\sum_{i=N}^{n} U_{i}\right)^{2} \leq B_{n}^{2} \leq 2 \mathbb{K} \max \left\{B_{n}, \sum_{i=N}^{n} x_{i}\right\}$ where

$\mathbb{K}=\sum_{j=\mathbb{N}}^{\infty} \mathcal{R}_{j}, \mathcal{R}_{n}=\sum_{i=\mathbb{N}}^{n} x_{i}$. Hence, we

have

$B_{n} \leq$

$\max \left\{2 \mathbb{K}_{,}\left(\left(2 \mathbb{K} \sum_{i=N}^{n} r_{i}\right)^{\frac{1}{2}}\right)\right\}$

It follows from $(U 1)$ and $(21)$ that $\lim _{n \rightarrow \infty} \frac{1}{n} B_{n}=0$ so that $\lim _{n \rightarrow \infty} \frac{1}{n} \sum_{i=\mathbb{N}}^{n}-u_{i+1}=0$.
Corollary 3.2 Let (U 1) hold. Then (1) is oscillatory in case (1)ither of the following holds

$$
-\infty<\inf \frac{1}{n} \sum_{i=0}^{n} \sum_{i=\mathbb{N}}^{\mathbb{d}} q_{\mathrm{i}}<\lim _{n \rightarrow \infty} \sup \frac{1}{n} \frac{1}{n} \sum_{\mathbb{i}=0}^{n} \sum_{\mathrm{i}=0}^{\mathbb{1}} q_{\mathrm{i}}
$$

(or)

$$
\lim _{n \rightarrow \infty} \frac{1}{n} \sum_{i=0}^{n} \sum_{i=0}^{1} q_{i}=+\infty
$$

Remark 3.3The Criteria given in corollary (3.2) are discrete analogues of the oscillation criteria of Hartman [Dosly and Rehak, 1998a] and wintner [Taylor, 1993] for equation (3) with $r(\epsilon) \equiv 1$

Theorem 3.4 (i) Let $(U 1)$ and (9) holds. Then if (1) is non oscillatory we can define the constant

$$
\psi \equiv \lim _{n \rightarrow \infty} \frac{1}{n} \sum_{i=0}^{n} \sum_{i=0}^{\mathbb{1}} q_{i}
$$

$$
u_{n}=\psi-\sum_{i=0}^{\text {such that }} \sum_{n=1}^{n-1} q_{i}+\sum_{i=n}^{\infty} \frac{u_{i}^{2}}{u_{i}+r_{i}^{2}} \geq 0 ; n \geq \mathbb{N}
$$

(16ii) If there exists a sequence $u$, un $>-r n, n \geq N \geq 0$ and $a$ constant $\psi$ such that

$$
u_{n} \geq \psi-\sum_{i=0}^{\infty} \sum_{i}+\sum_{i=n}^{\infty} \frac{u_{i}^{2}}{u_{i}+r_{i}^{2}} \geq 0
$$

(or)

$$
\begin{aligned}
& u_{n} \leq \psi-\sum_{i=0}^{n-1} \sum q_{i}+\sum_{i=n}^{\infty} \frac{u_{i}^{2}}{u_{i}+r_{i}^{n}} \leq 0(26) \\
& \text { then(1) is non oscillatory. }
\end{aligned}
$$

Theorem 3.5 Let (U 2) hold. If the sequence q satisfies

$\liminf _{n \rightarrow \infty} \frac{1}{n} \sum_{i=0}^{n} \sum_{i=0}^{\mathbb{1}} q_{i}=-\infty$

and

$\limsup _{n \rightarrow \infty} \frac{1}{n} \sum_{i=0}^{n} \sum_{i=0}^{1} q_{i}>-\infty$

then(1) is oscillatory.

Proof: suppose to the contrary that (1) is non oscillatory and let $U n=r n \Delta x n / x n$ for $n \geq 0$. Now since condition $(U 1)$ follows from ( $U 2)$, theorem (3.1) and (3.4) imply

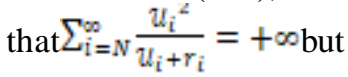

$\limsup _{n \rightarrow \infty} \frac{1}{n} \sum_{i=\mathbb{N}}^{n}\left(-u_{i+1}\right) \geq \liminf _{n \rightarrow \infty} \frac{1}{n} \sum_{i=n}^{\infty} \frac{u_{i}^{2}}{U_{i}+r_{i}^{n}}+\limsup _{n \rightarrow \infty} \frac{1}{n} \sum_{i=\mathbb{N}}^{n} \sum_{i=\mathbb{N}}^{\mathbb{1}} q_{i}$

which is impossible from 
$-u j+1<r j+1$. This completes the proof.

Theorem 3.6 Let (U 3) hold and assume (1) is non oscillatory. Then the following statements are equivalent

(i) $q \in \mathbb{C}$

(ii) $\lim _{n \rightarrow \infty} \frac{1}{-1} \sum_{k=0}^{n} \sum_{j=0}^{k} q_{j}$ exist (finite)

(iii) The sequence q satisfies (9)

(iv) For any non oscillatory solution $x$, with $x$ nxn $+1>0 . n \geq \mathbb{N}$ for some $\mathbb{N} \geq 0$, the sequence $x n=r n \Delta x n / x n_{s}$ satisfies $(10)$.

Proof: Obviously $($ i $) \Rightarrow($ ii $) \Rightarrow($ iii). Theorem (3.1) shows that (iii) and (iv) are equivalent. The proof of $(i v) \rightarrow(i)$ in immediate by letting $n \rightarrow \infty$ and observing that (iv) implies that $u n \rightarrow 0$ as $n \rightarrow \infty$.

This completes the proof.

Remark 3.7We observe therefore that theorem (3.6) show that if (U 3) holds and (1) is non oscillatory then the sequence q either converges or diverges to $-\infty$. This is not true in the differential equations case (cf [Tipler, 1978]). The following Oscillation tests are immediate.

Corollary 3.8 Let (A3) and (9) hold. If $q \notin \mathcal{C}$ then (1) is oscillatory.

Corollary 3.9 Let (A3) hold. If

$$
-\infty=\lim _{n \rightarrow \infty} \inf \sum_{j=0}^{n} q_{j}<\lim _{n \rightarrow \infty} \sup \sum_{j=0}^{n}
$$

Then (1) is oscillatory.

Theorem 3.10 If there exist two sequence $\{N k\}$ and $\{n k\}$ of integers $n k \geq N k+1$ such that

$N k \rightarrow \infty$ as $K \rightarrow \infty$ and

$\sum_{j=N_{k}}^{n_{k}-1} q_{j} \geq r_{N_{k}}+r_{n_{k}}$

Then (1) in oscillatory.

\section{CONJUGACY CRITERIA}

Theorem 4.1 Suppose that $q k \neq 0$ and $\sum_{j=-\infty}^{\infty} q_{j}=\lim _{k \rightarrow \infty} \sum_{j=-k}^{k} q_{j}$ exists (as a finite number). If

$$
\sum_{j=-\infty}^{\infty} q_{j} \geq 0
$$

equation (1) is conjugate on $\mathbb{Z}$.

Proof: Suppose for the contrary, that equation (1) is not conjugate on $\mathrm{Z}$. Then it is disconjugate and hence there exists a solution $x$ of (1) such that $r k x k x k+1>$ for all

$k \in$. We can assume that there exists a solution $w$ of the generalized Riccati difference equation with $r k+w k>$,

$k \in$, which is related to (1) by $w k=r k \varphi(\Delta y k / y$. Let $m, n$ be integers such that $0 \leq m<n$. $w_{n}-w_{m}=\sum_{j=m}^{n-1} q_{j}-\sum_{j=m}^{n-1} s\left(w_{j}, x_{j}\right)$

$w_{-m}-w_{-n}=\sum_{j=-n}^{m-1} p_{j} \sum_{j=m}^{n-1} s\left(w_{j}, x_{j}\right)$

Letting $n \rightarrow \infty$ and putting $m=0$ in the above equation we have

$-w_{0}=-\sum_{j=0}^{\infty} q_{j}-\sum_{j=0}^{\infty} s\left(w_{j}, x_{j}\right)$

and

$w_{0}=-\sum_{j=-\infty}^{-1} p_{j}-\sum_{j=-\infty}^{-1} s\left(w_{j}, r_{j}\right)$

Since $w k$ and also $w k$ and also $w-k$ tend to zero as $k \rightarrow \infty$ from the addition of last two inequalities we have

$\sum_{j=-\infty}^{\infty} q_{j}=-\sum_{j=-\infty}^{\infty} s\left(w_{\tilde{p}} r_{j}\right)$

which is a contradiction to (32).

Corollary 4.2 Let $m 1, m 2 \in \mathrm{N}$ be such that $m 2 \geq m 1+2$. A sufficient condition for (1) to be conjugate on the interval $[m 1, m 2]$ is that either

$\sum_{j=m_{1}} q_{j} \geq r_{m m_{1}}+r_{m m_{2}+1}$

(or)

$q_{m_{\mathrm{R}}} \geq r_{m m_{\mathrm{R}}}+r_{m \mathrm{~m}_{\mathrm{R}}+1}, i=1,2,3, \ldots$

q $q_{\left.\text {Theorem } 4.3 \text { Suppose that }{ }^{3} k\right)} \geq 0$ for $k \geq$. A sufficient condition for conjugacy of (1) in an interval $[n,+\infty), \quad n \in$, is that there exist integers $l$, $m$ with $n<l<m$ such that $\frac{1}{(1-n)^{\alpha-1}}<\sum_{k=1}^{m} q_{k}$

Proof: We will show that the solution $x$ of (1) given by the initial condition $x n=0, x n+1=1$ has a generalized zero in $(n, \infty)$. Then without loss of generality we can assume $x k>0$ in $(n, \infty)$ and $\Delta x k \geq 0$ in $[n, \infty)$, since if $\Delta x k<0$ at some point in $(n, \infty)$, we would have a generalized zero in $(n, \infty)$ by the condition $q k \geq 0$.

$\left(\Delta x_{m}\right)^{\alpha-1}=\left(\Delta x_{1}\right)^{\alpha-1}-\sum_{k=1}^{m}\left(x_{k}\right)^{\alpha-1} q_{k}$.

Since $q k \geq 0$, using the discrete version of the lagrange mean value theorem we have,

$\left(\Delta x_{1}\right)^{\alpha-1}-\sum_{k=1}^{m}\left(x_{k}\right)^{\alpha-1} q_{k} \leq\left(\Delta x_{1}\right)^{\alpha-1}-\sum_{k=1}^{m}\left(x_{1}\right)^{\alpha-1} q_{k}=\left(\Delta x_{1}\right)^{\alpha-1}[1$

By hypothesis, the factor in brackets is negative. If $\Delta x_{\mathbb{1}}>0$, then $\Delta x_{m+1}<0$, implying a generalized zero in $(m+1, \infty)$.

$x$ has a generalized zero in $(m+1, \infty)$ and so (1) in conjugate in $[n, \infty)$.

Remark 4.4The results 
presented in preposition (2.2) and lemma (2.3) are applied to check the conjugacy criteria for (1).

\section{CONCLUSION}

Here the complete study made on the discrete oscillation and conjugacy criteria using Riccati techniques. The research could be further extended in future for oscillation of higher order non linear as well as linear difference equation.

\section{ACKNOWLEDGMENT}

The author K.ShirmilaPremkumari thanks the co-author Dr.Daphy Louis Lovenia for encour- aging to write this research paper and also I would like to thank all of them for given their cooperation to do the research.

\section{REFERENCES}

1. [Chen and Erbe, 1989] Chen, S. and Erbe, L. H. (1989).Riccati techniques and discrete oscil- lation.J. Math. Anal.Appl, 88(142):468-487.

[Cheng, 1994] Cheng, S. S. (1994). Hille-WintnerComposision Theorems for Non Linear diff.eqn. Funkcial, EKvac, 88(37):531-535.

2. [Dosly and Rehak, 1998a] Dosly, O. and Rehak, P. (1998a). Conjugacy Criteria for SecondOrder Lineardiff.eqn. Arch. Math., 88(34):301-319.

3. [Dosly and Rehak, 1998b] Dosly, O. and Rehak, P. (1998b). Non Oscillatorn Criteria for Sec- ond Order Lineardiff.eqn. Arch. Math. 88(34):301-319.

4. [Erbe and Yan, 1992] Erbe, L. H. and Yan, P. (1992). Weighted Averaging Techniques in Oscillation Theory for Second Order diff.eq.Canad Math. Bull., 88(35):61-69.

5. [Hooker et al., 1987] Hooker, J. W., Kwong, M. K., and Patula, W. T. (1987). Oscillatory Second Order Linear diff.eqn.andRiccati equation,. SIAM. J. Math. Anal., 88(18):54-63.

6. [Hooker and Patula, 1981] Hooker, J. W. and Patula, W. T. (1981).Riccati Type Transfor- mations for Second-Order Linear diff.eqn.J. Math. Anal.Appl, 88(82):451-462.

7. [Kwong et al., ]Kwong, M. K., Hooker, J. W., and Patula, W. T. Riccati Type Transformation for Second-order linear diff. eqn,.J. Math. Anal. Appl., 88(107):182-196.

8. [Mary, 1987] Mary, D. F. S. (1987). RiccatiIntesral equations and non oscillation of self-adjoint linear systems.J. Math. Anal.Appl, 88(121):109-118.

9. [Patula, 1979] Patula, W. T. (1979). Growth and Oscillation Properties of Second Order linear diff. eqns. SIAM. J. Math. Anal., 88(10):1272-1279.

10. [Selvaraj and Daphy, 2011] Selvaraj, B. and Daphy, J. (2011). Oscillatory Properties of Cer- tain First and Second order Diff. eqn. $J$. Comp and Math. aci. val., 88(2(3)):567-571.

11.

Selvaraj and Lovenia, ]Selvaraj, B. and Lovenia, J. D. L. Third-order newtral difference equations with positive and negative co-esticients! $J$. Comp. Math. Sci., 88(2(3)):531-536.

12. [Taylor, 1993] Taylor, W. E. (1993). Fourth order diff. eqn, Oscillation and non oscillation. Rocky Mountain J. Math, 88(23):781-795.

13. [Tipler, 1978] Tipler, F. J. (1978). General relativity and Conjugate ordinary differential eqn. J. Differential equation, 88(30):165-174.

14. [Wintner, 1951] Wintner, A. (1951). On the non existence of conjugate points.Amer. J. math, 88(73):368-380.

\section{AUTHORS PROFILE}

ShirmilaPremkumari. K, Karunya Institute of Technology and Sciences, Coimbatore, India.abiramidarshan74@gmail.com. 